Editorial

\title{
New Frontiers for Information and Communication Technologies in Scientific Publishing
}

\author{
Filippo Boniforti ${ }^{1}$ Giuseppe Milano ${ }^{2}$ \\ ${ }^{1}$ Fondazione Ospedale Giglio, Cefalù, Italy \\ ${ }^{2}$ Department of Orthopaedics, Catholic University, Rome, Italy
}

Joints 2018;6:1.

Communicating, conveying information, and transferring knowledge, these are actions that all living beings engage in, and they are central to scientific activity. Communication strategies involve the use of well-codified techniques and rules, and scientific communication, despite the incredible changes and innovations ushered in by the digital age, continues to hinge on the written word. However, the passing of time has brought changes in the methods used for transferring information, also as an effect of their scientific verification in terms of efficacy and safety. It has been shown, for example, that airline passengers understand safety maneuvers better if the instructions they receive are supported by illustrations.

The communicative potential of images has also been exploited by health care professionals in hospitals, with this approach being found to be extremely useful in conveying information about medical and surgical procedures not just to patients, but also among physicians and other health care professionals. Furthermore, the Internet, the use of icons to support text, touchscreens, and digitization, have created many different information/communication opportunities, all complementary to the use of written text.

Infographics have become, in form and stature, a valid scientific communication strategy. In particular, more and more people, drawn to the use of graphics in association with scientific data, are finding that infographic techniques suit them better.

The use of graphics reinforces visual memory; recollection of a message is extremely increased if it is conveyed through text and images as opposed to text alone. Furthermore, the diffusion of images digitally makes it possible, thanks to the use of social media, to reach a wider audience and achieve greater popularity, in terms of both visualizations and cita- tions. Nowadays, sharing via Twitter, Facebook, or Instagram must be considered an option for transferring information, also in the scientific field. Although an image is obviously no substitute for reading a complete research paper, it adds visual value to the text, serving to illustrate the key concepts. The National Institute for Health and Care Excellence, for example, used an infographic series to spread guidelines in orthopaedics and traumatology and validated this methodology.

Like all techniques, infographics have their own rules, and although, they allow the reader to obtain an opinion rapidly, the design work involved is time consuming. As far as our specific sphere is concerned, infographics are designed by physicians (who must have an active role in the image preparation process) and are aimed at physicians. That said, any one of us, provided we are properly supported by adequate technology and dedicated software, can produce an attractive infographic that meets several key requirements: the recipient of the message (target) must be clearly established; the title must be clear, and it must contain the key message of the scientific work that is the subject of the infographic; arrows and lines must be used to guide the reading process in a logical and orderly manner; spatial proportions between images, drawings, and words must be appropriate, and there needs to be a harmonious balance in the use of colors and fonts.

We felt it would be interesting to make our readers aware of this method of information/communication that, by combining images with words in a new way, may facilitate recall of data, concepts, and recommendations, or even just rapid dissemination of critical ideas. Researchers wanting to share their work with the scientific community are informed that, from now on, Joints is willing to include works prepared according to this new publishing format.
Address for correspondence Giuseppe Milano, MD, Department of Orthopaedics, Service of Shoulder Surgery,

A. Gemelli University Hospital, Largo Agostino Gemelli, 00136, Rome, Italy

(e-mail: giuseppe.

milano@unicatt.it).
DOI https://doi.org/

10.1055/s-0038-1641718. ISSN 2282-4324.
Copyright (C) 2018 Georg Thieme Verlag License terms KG Stuttgart - New York 